\title{
Heart rate elevations during early sepsis predict death in fluid-resuscitated rats with fecal peritonitis
}

Alain Rudiger ${ }^{1 *} \mathbb{D}$, Victor Jeger ${ }^{1,2}$, Mattia Arrigo ${ }^{3}$, Christian A. Schaer ${ }^{1,2}$, Florian F. Hildenbrand ${ }^{2}$, Margarete Arras ${ }^{4}$, Burkhardt Seifert ${ }^{5}$, Mervyn Singer ${ }^{6}$, Gabriele Schoedon², Donat R. Spahn ${ }^{1}$ and Dominique Bettex ${ }^{1}$

* Correspondence: alain.rudiger@ usz.ch

${ }^{1}$ Institute of Anesthesiology, University and University Hospital Zurich, Raemistrasse 100, 8091 Zurich, Switzerland

Full list of author information is available at the end of the article

\section{Abstract}

Background: In sepsis, early outcome prediction would allow investigation of both adaptive mechanisms underlying survival and maladaptive mechanisms resulting in death. The aim of this study was to test whether early changes in heart rate monitored by telemetry could predict outcome in a long-term rat model of fecal peritonitis.

Methods: Male Wistar rats $(n=24)$ were instrumented with a central venous line for administration of fluids, antibiotics and analgesics. A telemetry transmitter continuously collected electrocardiogram signals. Sepsis was induced by intraperitoneal injection of fecal slurry, and the animals were observed for $48 \mathrm{~h}$. Additional animals underwent arterial cannulation at baseline $(n=9), 4 \mathrm{~h}(n=16)$, or $24 \mathrm{~h}(n=6)$ for physiology and laboratory measurements.

Results: 48-h mortality was 33\% (8/24), with all deaths occurring between 4 and 22 h. Septic animals were characterized by lethargy, fever, tachycardia, positive blood cultures, and elevated cytokine (IL-1, IL-6, TNF alpha) levels. An increase in heart rate $\geq 50$ bpm during the first $4 \mathrm{~h}$ of sepsis predicted death with sensitivity and specificity of $88 \%(p=0.001)$.

Conclusions: In this long-term rat sepsis model, prognostication could be made early by telemetry-monitored changes in heart rate. This model enables the study of underlying mechanisms and the assessment of any differential effects of novel therapies in predicted survivors or non-survivors.

Keywords: Sepsis, Animal model, Fecal peritonitis, Telemetry, Electrocardiogram, Heart rate, Outcome

\section{Background}

Sepsis, the dysregulated host response to infection leading to organ dysfunction, is a common and frequently fatal condition [1], with as many annual deaths as those from acute myocardial infarction [1,2]. Despite major efforts over the last decade, mortality rates still range between 25 and $50 \%$ [1, 3, 4]. Hence, a better understanding of the underlying pathophysiology and new therapeutic concepts are urgently needed to improve outcome. To date, mechanistic studies have been predominantly performed with comparison against non-septic control (sham) animals. However, this approach has the important limitation of not discriminating between adaptive mechanisms of survival

(c) The Author(s). 2018 Open Access This article is distributed under the terms of the Creative Commons Attribution 4.0 International License (http://creativecommons.org/licenses/by/4.0/), which permits unrestricted use, distribution, and reproduction in any medium, provided you give appropriate credit to the original author(s) and the source, provide a link to the Creative Commons license, and indicate if changes were made. 
and maladaptive mechanisms of death. Early prediction of outcome in an individual animal would enable such discrimination. It would also greatly facilitate the testing of novel therapies to determine any survival impact in likely non-survivors while focusing on safety and the rate/degree of recovery in likely survivors.

We previously reported that echocardiography-derived stroke volume and heart rate could prognosticate as early as $3-6 \mathrm{~h}$ in a 3-day rat model of fecal peritonitis [5]. Echocardiography however requires anesthesia, expensive equipment, and experienced investigators. Heart rate can also be measured in real-time by telemetry, with continuous monitoring of the electrocardiogram (ECG) in awake animals. In addition, ECG telemetry provides information on heart rate variability and documents the precise time of demise. The aim of the current study was to test whether telemetry-monitored changes in heart rate during early sepsis could predict outcome in a long-term (48 h) rat model of fecal peritonitis. For clinical relevance, this model received fluid resuscitation and antibiotics, and analgesia to meet the high requirements of animal welfare.

\section{Methods}

\section{Animal model}

All animal experiments were performed in the animal laboratories of the University Hospital Zurich, Switzerland. All protocols were approved by the Animal Experimentation Committee of the Veterinary Office of the Canton of Zurich, Switzerland. Principles of the 3Rs were implemented [6,7].

The current animal model has been adapted from a model of fecal peritonitis, which is established and still being in use at University College London, UK [5, 8, 9]. Figures 1 and 2 summarize the timelines and numbers of animals (n) for each group. A total of 61 animals were included in this study ( $48 \mathrm{~h}$ outcome study: sepsis $n=24$ and sham $n=6$; physiology and laboratory measurement study: baseline $n=9 ; 4 \mathrm{~h}$ sepsis $n=16$; 24 h sepsis $n=6$ ).

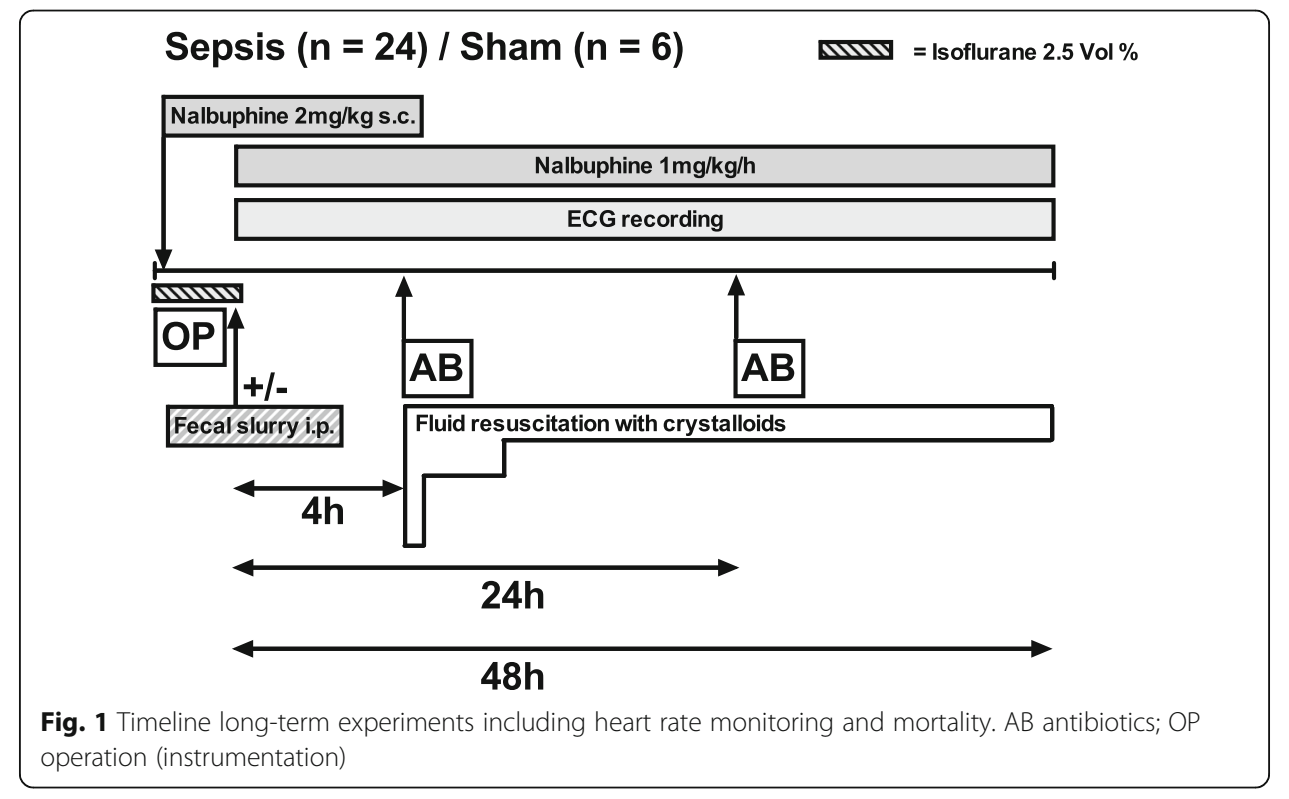




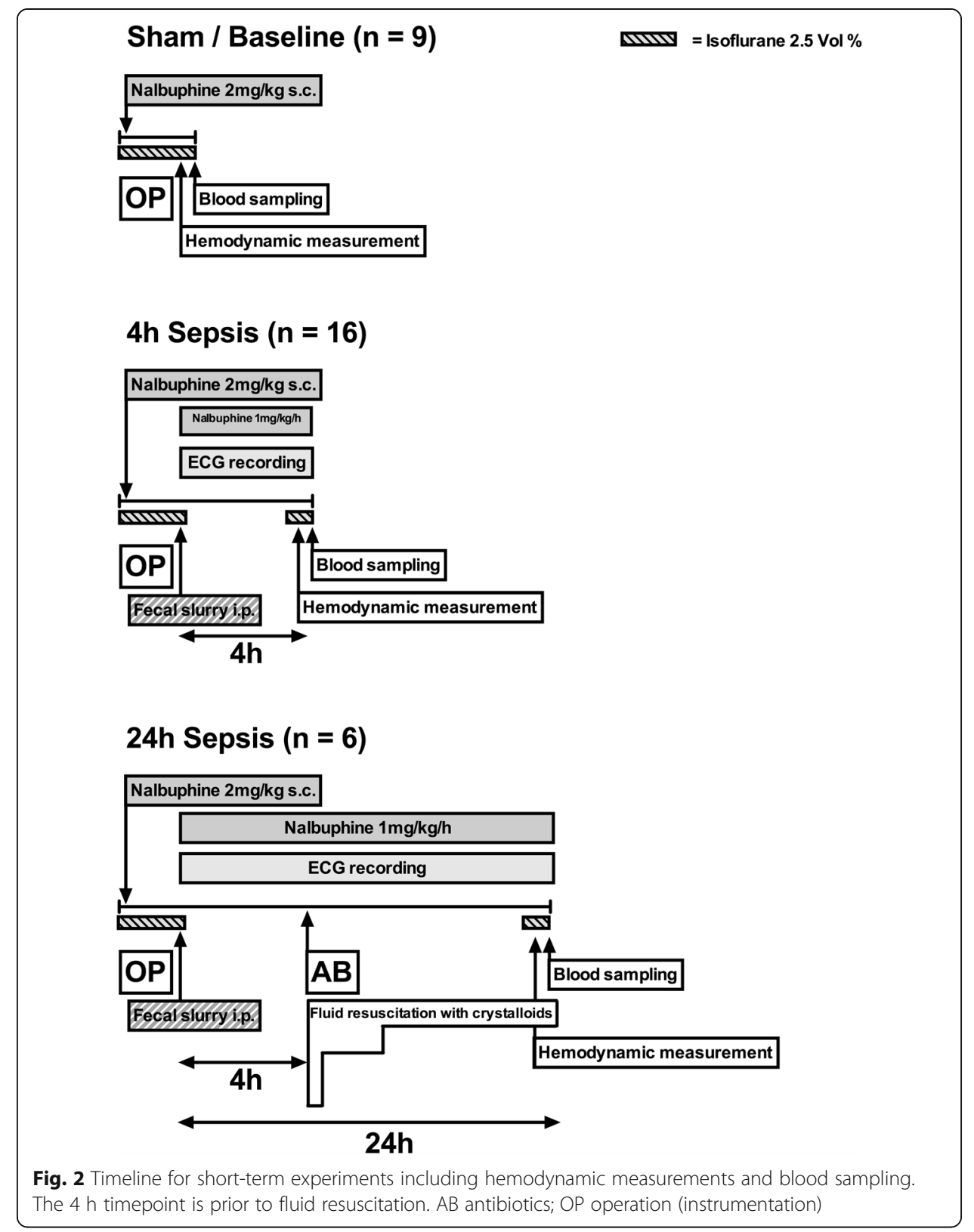

Under general anesthesia (2.5\% isoflurane in room air), male Wistar rats were instrumented with a central venous line and a swivel-tether system allowing the rat, after recovery from anesthesia, to have unimpeded movement in its cage. Details on instrumentation have been reported previously [10].

The animals received the opioid analgesic nalbuphine subcutaneously prior to instrumentation $(2 \mathrm{mg} / \mathrm{kg})$ and as a continuous infusion thereafter $(1 \mathrm{mg} / \mathrm{kg} / \mathrm{h})[10]$. The rationale for the use of nalbuphine has been summarized recently [11].

While still under anesthesia, sepsis was induced by an intraperitoneal injection of $2 \mathrm{~mL} /$ $\mathrm{kg}$ fecal slurry (25\% suspension). Fecal slurry contained feces collected from several animals of the same batch that was suspended in Ringer's acetate, and then filtered. Therefore, our model has two interventions, namely, surgery (comprising cannulation of the internal jugular vein and subcutaneous implantation of the telemetry device), and 
injection of fecal slurry into the peritoneal cavity immediately thereafter. Sham animals received fluids and nalbuphine, but did not receive any intraperitoneal injection.

Fluid resuscitation with Ringer's acetate was commenced $4 \mathrm{~h}$ after the septic insult through the central venous line. After a fluid challenge of $20 \mathrm{~mL} / \mathrm{kg}$ given over $15 \mathrm{~min}$, crystalloids were infused at a rate of $10 \mathrm{~mL} / \mathrm{kg} / \mathrm{h}$ between 4 and $8 \mathrm{~h}$. At $8 \mathrm{~h}$, the infusion rate was reduced to $5 \mathrm{~mL} / \mathrm{h}$, and at $24 \mathrm{~h}$ halved again to $2.5 \mathrm{~mL} / \mathrm{h}$. This fluid resuscitation protocol was adapted from an established animal model $[5,8]$ by replacing the $1: 1$ solution of $6 \%$ hetastarch and glucose with crystalloids and reducing the overall amount of fluids administered.

Ceftriaxone $30 \mathrm{mg} / \mathrm{kg}$ was given intravenously 4 and $24 \mathrm{~h}$ after the septic insult [12]. To confirm that our animals developed clinically relevant infection, two additional animals were instrumented as described above, and received fecal slurry but no antibiotics. $6 \mathrm{~h}$ after sepsis induction, the blood and peritoneal fluids were sampled for microbiological analyses performed at the Institute for Medical Microbiology, University Zurich.

Baseline values of heart rate were collected 15 min after the end of instrumentation, when the animal had recovered from general anesthesia. Observation time in the long-term experiments was $48 \mathrm{~h}$. Time of death was defined by asystole in the ECG.

For physiology and laboratory measurements, animals were prepared as described above (Fig. 2). At baseline, $4 \mathrm{~h}$ (prior to fluid resuscitation) or $24 \mathrm{~h}$, a catheter was placed in the right carotid artery under isoflurane anesthesia, which allowed blood pressure measurements and arterial blood sampling. The arterial line was attached to a pressure transducer (ADInstruments, Oxford, UK), as previously described [10].

Blood gas analysis was performed with an Epoc blood analyzer (Epocal Inc. Ottawa, Ontario, Canada). Plasma cytokines were analyzed with a Bio-Plex Pro Rat cytokine 24-plex assay using the Bio-Plex 200 Suspension Array System (Bio-Rad Laboratories AG, Cressier, Switzerland). B-type natriuretic peptides (BNP) and troponin ITC complex were measured using the Rat BNP and Rat Troponin ITC Complex Assay kits (MesoScale Discovery, Rockville, MD, USA) according to the manufacturers' instructions.

\section{Heart rate measurements}

Under general anesthesia, a telemetry transmitter (Model RT50B, Millar, Houston, TX, USA) was implanted subcutaneously under the xyphoid process. Two electrodes were placed subcutaneously to either side of the chest, as previously described [10]. No prophylactic antibiotics were given for this procedure.

ECG signals were collected by a SmartPad TR181 (Millar) at a sampling rate of $1 \mathrm{kHz}$ and then transferred via a PowerLab (ADInstruments) to a PC. Heart rate and heart rate variability were analyzed with LabChart V7 (ADInstruments) by a blinded investigator (MA) using published guidelines [13]. Ectopic beats and artifacts were excluded from analysis. R-R intervals were classified as normal (NN) between 100 and $250 \mathrm{~ms}$. A NN difference $>50 \mathrm{~ms}$ was defined as the variation threshold. Settings included a FFT size of 1024 and a window according to Hann [14]. We focused our analysis of HRV on SDNN (standard deviation of normal-normal $(\mathrm{NN})$ intervals (=R-R intervals); reflecting cyclic components of sympathetic and parasympathetic activity); total power, the variance of all NN intervals; and the ratio of LF/HF, where LF represents the power of low-frequency range (sympathetic response) and HF the power of high-frequency range (parasympathetic response) [14]. 


\section{Statistics}

All results are indicated as mean \pm standard deviation (SD) with the exception of Figs. 4 and 5 where standard error of the mean (SEM) was used to enhance readability. Assuming a sepsis mortality of $25-50 \%$ in the long-term study, a sample size of 24 septic animals was calculated to have at least six animals in the group of non-survivors. In the short-term experiments, a minimum of five animals was accepted in the group of predicted non-survivors in order to limit the number of animals suffering from a high severity of illness. Comparisons between groups were analyzed with ANOVA and Tukey's post hoc test, as appropriate. For data in Table 1, cytokines and cardiac

Table 1 Physiological and laboratory variables

\begin{tabular}{|c|c|c|c|c|c|}
\hline \multirow[t]{2}{*}{ Variables } & \multirow{2}{*}{$\begin{array}{l}\text { Sham, } \\
\text { baseline } \\
n=9\end{array}$} & \multicolumn{2}{|l|}{$\begin{array}{l}\text { Sepsis, } 4 \text { h } \\
n=16\end{array}$} & \multirow{2}{*}{$\begin{array}{l}\text { Sepsis, } 24 \mathrm{~h} \\
n=6\end{array}$} & \multirow[t]{2}{*}{$\begin{array}{l}p \text { value } \\
\text { (ANOVA) }\end{array}$} \\
\hline & & $\begin{array}{l}\text { Predicted } \\
\text { survivors } \\
n=11\end{array}$ & $\begin{array}{l}\text { Predicted non- } \\
\text { survivors } \\
n=5\end{array}$ & & \\
\hline \multicolumn{6}{|l|}{ Physiological variable } \\
\hline Rectal temperature $\left[{ }^{\circ} \mathrm{C}\right]$ & $37.4(0.3)$ & $38.5(0.4)^{*}$ & $39.1(1.3)^{*}$ & $38.1(0.8)$ & 0.003 \\
\hline \multicolumn{6}{|l|}{ Hemodynamic variables } \\
\hline Heart rate $[1 / \mathrm{min}]$ & $346(39)$ & $383(44)$ & $444(91)^{*}$ & $418(41)$ & 0.012 \\
\hline $\begin{array}{l}\text { Change in heart rate from } \\
\text { baseline }[1 / \mathrm{min}]\end{array}$ & $\begin{array}{l}\text { not } \\
\text { applicable }\end{array}$ & $-71(53)$ & $78(24) \$$ & $-19(26)$ & $<0.001$ \\
\hline $\mathrm{MAP}[\mathrm{mmHg}]$ & $89(13)$ & $94(20)$ & $91(19)$ & $91(15)$ & 0.949 \\
\hline \multicolumn{6}{|l|}{ Arterial blood gases } \\
\hline $\mathrm{PaO}_{2}[\mathrm{kPa}]$ & $9.63(1.97)$ & $9.62(2.57)$ & $9.21(1.75)$ & $8.96(2.62)$ & 0.946 \\
\hline $\mathrm{PaCO}_{2}[\mathrm{kPa}]$ & $5.07(0.96)$ & $5.50(1.26)$ & $5.21(0.81)$ & $7.12(2.29)$ & 0.089 \\
\hline $\mathrm{pH}$ & $7.38(0.05)$ & $7.40(0.03)$ & $7.43(0.06)$ & $7.36(0.11)$ & 0.406 \\
\hline Base excess $[\mathrm{mmol} / \mathrm{L}]$ & $-2.6(4.7)$ & $0.6(5.4)$ & $0.7(3.0)$ & $2.7(2.4)$ & 0.302 \\
\hline Lactate $[\mathrm{mmol} / \mathrm{L}]$ & $1.0(0.3)$ & $1.2(0.2) 9$ & $1.6(0.7)$ & $1.9(0.8) £$ & 0.014 \\
\hline Glucose $[\mathrm{mmol} / \mathrm{L}]$ & $13.0(1.7)$ & $12.6(2.8)$ & $15.6(2.5)$ & $11.3(1.4)$ & 0.055 \\
\hline Hematocrit [\%] & $34(3)$ & $34(7)$ & $45(4)^{*} \$$ & $37(7)$ & 0.011 \\
\hline \multicolumn{6}{|l|}{ Cytokines (median, IQR) } \\
\hline Interleukin-1 beta [pg/mL] & $72(57-92)$ & $129(105-156)$ & $332(81-421) * 9$ & $58(19-91)$ & 0.001 \\
\hline Interleukin-6 [pg/mL] & $0(0-0)$ & $236(138-691)^{*}$ & $3062(134-8190)^{*}$ & $109(0-157)$ & $<0.001$ \\
\hline Interleukin-10 [pg/mL] & $116(59-149)$ & $182(155-493)$ & $573(114-1051)$ & $258(113-367)$ & 0.027 \\
\hline TNF alpha [pg/mL] & $33(0-49)$ & $93(63-154)^{*}$ & $135(47-166)$ & $35(0-37)$ & $<0.001$ \\
\hline $\mathrm{CXCL}-1[\mathrm{pg} / \mathrm{mL}]$ & $195(51-497)$ & $877(650-1492)^{*}$ & $1562(580-1773)^{*}$ & $339(111-454)$ & 0.001 \\
\hline MCP-1 [pg/mL] & $1.6(1.3-1.6)$ & $4.9(3.4-5.5)^{*}$ & $9.3(3.1-14.4)^{*}$ & $5.4(1.1-9.1)$ & 0.001 \\
\hline MIP-1 [pg/mL] & $8(8-14)$ & $50(15-150)^{*}$ & $262(71-474) * 9$ & $17(1-21)$ & $<0.001$ \\
\hline \multicolumn{6}{|l|}{ Cardiac biomarkers (median, IQR) } \\
\hline Troponin $[\mathrm{ng} / \mathrm{mL}]$ & $0.0(0.0-0.03)$ & $0.0(0.0-0.0)$ & $0.0(0.0-0.05)$ & $0.0(0.0-0.06)$ & 0.152 \\
\hline BNP $[\mathrm{pg} / \mathrm{mL}]$ & $0.4(0.2-0.8)$ & $0.3(0.2-0.5)$ & $0.0(0.0-0.3)$ & $1.0(0.3-2.5)$ & 0.099 \\
\hline
\end{tabular}

Values are shown as mean (SD), except for cytokines and cardiac biomarkers, where data were not normally distributed (median, interquartile range $=\mathrm{IQR}$ ). Groups were compared using ANOVA (for normally distributed data) or Kruskal-Wallis test (for not-normally distributed data) followed by according post hoc tests correcting for multiple testing. Significant group differences $(p<0.05)$ : *sham baseline versus sepsis $4 \mathrm{~h}$, \$predicted survivors versus non-survivors, 9 sepsis $4 \mathrm{~h}$ versus sepsis $24 \mathrm{~h}, £$ sham baseline versus $24 \mathrm{~h}$. BNP B-type natriuretic peptide; CXCL-1 chemokine (C-X-C motif) ligand 1, a neutrophil chemoattractant involved in clearance of bacterial infections; $M A P$ mean arterial pressure; $M C P-1$ monocyte chemoattractant protein-1, primarily secreted by activated monocytes and macrophages; MIP-1 macrophage inflammatory protein-1, chemotactic cytokine produced by macrophages upon endotoxin activation; TNF tumor necrosis factor 
biomarkers were not normally distributed and were therefore presented as median, interquartile range and tested using the Kruskal-Wallis test followed by post hoc test (Dunn) correcting for multiple testing. The quality of heart rate and its changes as diagnostic tests were described by the area under the ROC (receiver operator characteristic) curve (AUC). Fisher's exact test was used to compare positive and negative predictive values between groups. A $p$ value below 0.05 was considered significant, and all hypothesis testing was two-tailed. Prism 6 (GraphPad Software, La Jolla, CA, USA) was used to perform statistics and draw the figures.

\section{Results}

Animal model

Twenty-four septic animals (weight $396 \pm 73 \mathrm{~g}$ ) and 6 sham animals (weight $366 \pm 33 \mathrm{~g}$ ) were observed for $48 \mathrm{~h}$. Septic rats became lethargic and febrile as features of acute illness. Eight septic animals (33\%) died after $13.7 \pm 6.6 \mathrm{~h}$, with all deaths occurring between 4 and $22 \mathrm{~h}$ (Fig. 3). Animals surviving beyond $24 \mathrm{~h}$ showed signs of recovery with increased activity and improved interest in their surroundings, but developed edema and a bloated abdomen. Heart rate changes are displayed in Fig. 4. Results of the heart rate variability analysis are given in Fig. 5.

\section{Laboratory findings}

Microbiological cultures of ascites revealed gram-negative (Escherichia coli) and gram-positive (Enterococcus sp., alpha-hemolytic streptococci, Corynebacterium sp.) bacteria. Blood cultures were positive for E. coli, Enterococcus faecalis, and Staphylococcus sp. Antibiotic-resistance tests confirmed that the E. coli was sensitive to ceftriaxone.

Physiological and laboratory variables were measured in 9 sham and 22 septic animals. Results are displayed in Table 1.

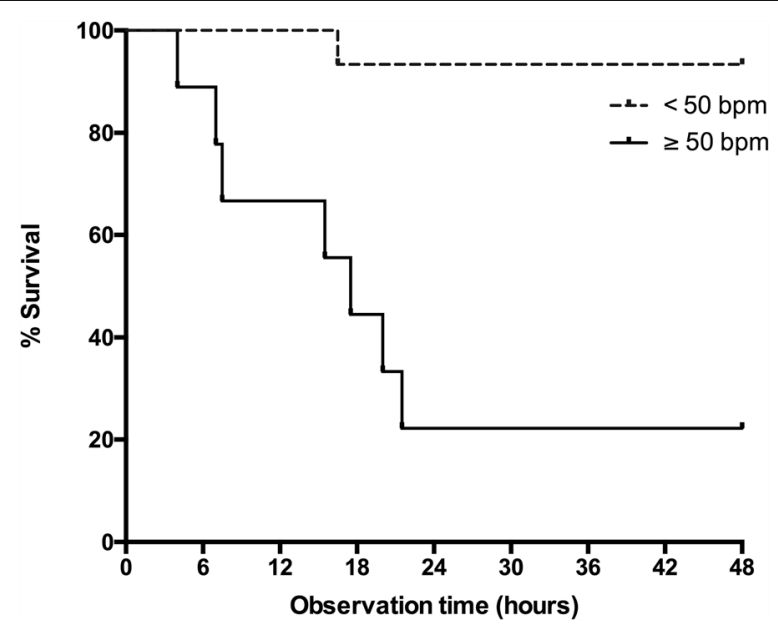

Fig. 3 Kaplan-Meier survival curves in septic animals according to the change in heart rate between baseline and $4 \mathrm{~h}$ of sepsis. Twenty-four septic Wistar rats were observed for $48 \mathrm{~h}$. Survival curves are shown for animals with a change in heart rate between baseline and $4 \mathrm{~h}$ of $<50 \mathrm{bpm}$ (dashed line; mortality $1 / 15=7 \%$ ) and those with a change $\geq 50 \mathrm{bpm}$ (continuous line; mortality $7 / 9=78 \%$ ), log rank $p<0.001$ 


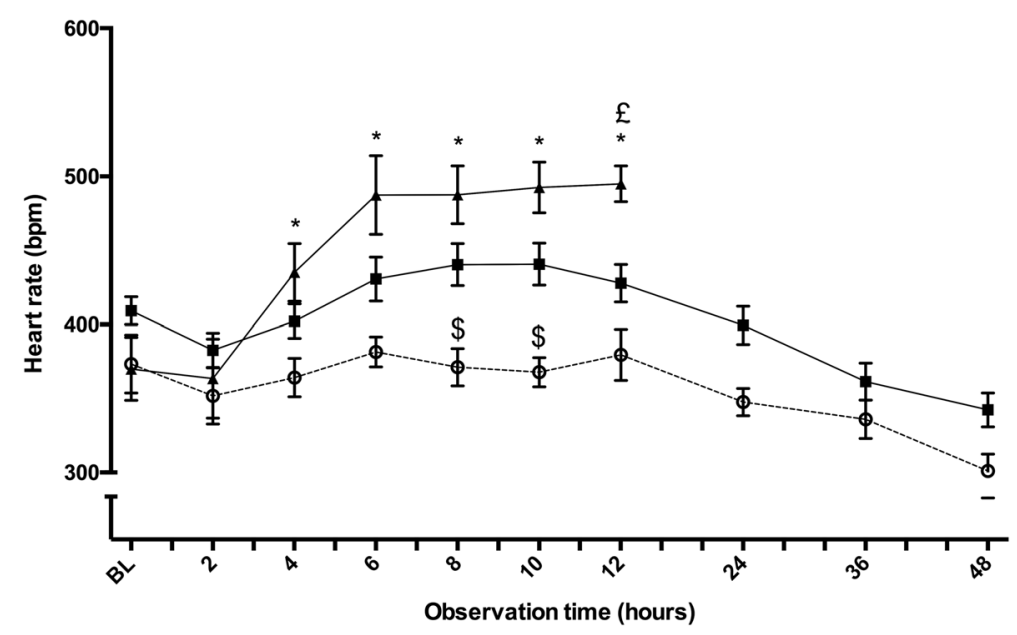

Fig. 4 Heart rate in septic animals. Temporal changes of telemetry-monitored heart rate after induction of sepsis in 8 non-survivors (black triangles), 16 sepsis survivors (black squares), and 6 sham animals (open circles). Baseline refers to the timepoint 15 min after slurry injection in septic animals (or 15 min after end of instrumentation in sham animals). Symbols indicate means (SEM). Significant group differences $(p<0.05)$ : *sham versus non-survivors, \$ sham versus survivors, $£$ survivors versus non-survivors. Data of non-survivors were only displayed until $12 \mathrm{~h}$, to avoid a selection bias due to mortality

\section{Prognosticators of outcome}

Compared to baseline values, changes in heart rate at 4 and $6 \mathrm{~h}$ were significantly different in sepsis survivors compared to non-survivors: $-7 \pm 48 \mathrm{bpm}$ vs $65 \pm 53 \mathrm{bpm}$ $(p=0.003)$ and $21 \pm 53 \mathrm{bpm}$ vs $120 \pm 61 \mathrm{bpm}(p=0.001)$, respectively. Prognosticators of death in septic animals are shown in Table 2. Importantly, an increase in heart rate $\geq 50 \mathrm{bpm}$ during the first $4 \mathrm{~h}$ of sepsis occurred in 7 of 8 non-survivors (sensitivity $88 \%$ ) and 2 of 16 survivors (specificity 88\%), Fisher's exact test $p=0.001$. Hence, the positive and negative predictive values for this heart rate cut-off level were 78 and $93 \%$, respectively.

Parameters from septic animals sacrificed at $4 \mathrm{~h}$ were tested for their prognostic potential to discriminate between predicted survivors and non-survivors (Table 3 ). Only hematocrit (AUC 0.93, 95\% CI 0.779-1.0, $p=0.008$ ) and MIP-1 (AUC 0.82, 95\% CI $0.604-1.0, p=0.047$ ) were statistically different between the two groups.

\section{Discussion}

We describe a clinically relevant rat model of sepsis with a $48 \mathrm{~h}$ mortality rate of $33 \%$. The animals developed a typical sepsis phenotype with clinical signs of illness (reduced activity, fever), tachycardia, elevated plasma cytokine levels, positive blood cultures, and peritonitis on postmortem examination. All deaths occurred between 4 and $22 \mathrm{~h}$ while animals surviving beyond this range showed clear signs of clinical recovery. Heart rate changes during early sepsis were prognostic for $48 \mathrm{~h}$ mortality. At $4 \mathrm{~h}$ an increase in heart rate $\geq 50 \mathrm{bpm}$ over baseline predicted mortality with positive and negative predictive values of $78 \%$ and $93 \%$, respectively.

The current sepsis model is based upon a well-established rat model of fecal peritonitis $[5,8]$ but to which antibiotics and a continuous infusion of the potent opioid analgesic, nalbuphine $[10,11]$ have now been added. Injection of intra-peritoneal fecal slurry in non-antibiotic-treated animals results in polymicrobial sepsis with positive 

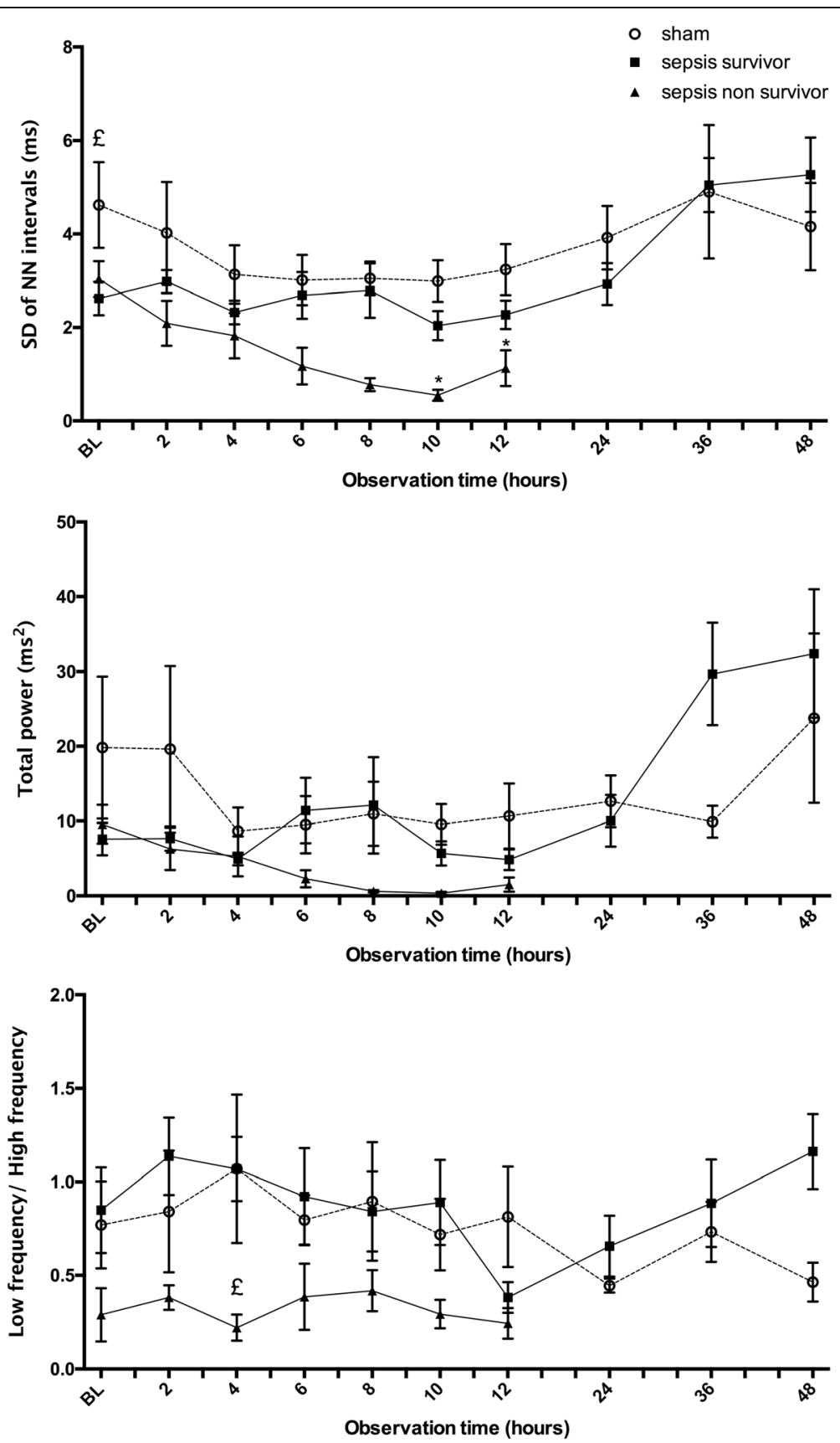

Fig. 5 Heart rate variability in septic animals. Baseline refers to the timepoint 15 min after slurry injection in septic animals (or 15 min after end of instrumentation in sham animals). In the top figure, temporal changes of the standard deviation (SD) of normal-to-normal (NN) inter-beat intervals are depicted. Total power (TP) is shown in the middle figure. Both represent global parameters of HRV. The bottom figure shows the ratio between low frequency (LF) and high frequency (HF), reflecting the relationship between sympathetic and parasympathetic activity. Symbols indicate means (SEM). Eight non-survivors (black triangles), 16 survivors (black squares), and 6 sham animals (open circles).Significant group differences $(p<0.05)$ : *sham versus non-survivors, $£$ survivors versus non-survivors

blood cultures, while post-mortem examination demonstrates generalized peritonitis and ascites formation. Injection of fecal slurry allows an identical insult in a batch of animals while avoiding a laparotomy reduces surgical trauma and postoperative pain. 
Table 2 Prognosticators of death in septic animals

\begin{tabular}{llll}
\hline Physiological variables & ROC AUC & $95 \% \mathrm{Cl}$ & $p$ value \\
\hline HR at $4 \mathrm{~h}(n=24)$ & 0.664 & $0.409-0.919$ & 0.198 \\
$\mathrm{HR}$ at $6 \mathrm{~h}(n=23)$ & 0.732 & $0.507-0.957$ & 0.082 \\
HR change between baseline and $4 \mathrm{~h}(n=24)$ & 0.875 & $0.684-1.00$ & 0.003 \\
HR change between baseline and 6 $\mathrm{h}(n=23)$ & 0.879 & $0.681-1.00$ & 0.005 \\
\hline
\end{tabular}

AUC area under the curve, $C l$ confidence interval, $h$ hours, $H R$ heart rate, $R O C$ receiver operator characteristic. By $6 \mathrm{~h}$, one septic animal had died and could not be used for ROC calculations

Septic animals developed an early tachycardia that progressed, especially in eventual non-survivors, notwithstanding aggressive intravenous fluid resuscitation commencing from $4 \mathrm{~h}$. Mechanisms such as sympathetic overstimulation are likely present, despite the use of continuous opioid analgesia to manage pain. Afferent fibers in the periphery can sense inflammation and directly activate autonomic centers within the brainstem [15]. This could be particularly important with peritonitis as the region around the celiac axis has a rich supply of sympathetic afferents [15].

The prognostic finding of tachycardia mirrors findings from patients with both non-septic [16] and septic [17-20] conditions. In our current study, an increase in heart rate $\geq 50 \mathrm{bpm}$ during the first hours of sepsis strongly prognosticated a poor outcome. Our data support the hypothesis that outcome is determined at an early stage [21]. In our model, using an identical insult in animals of similar genotype, age, sex and upbringing, this was true even before commencement of fluid resuscitation and antibiotics. Hence, predicted non-survivors did not benefit from these standards of care, highlighting the need for new therapeutic concepts to improve survival in this group. The risk for predicted survivors is harm from additional interventions [22]; this may result in no overall benefit for the sepsis population as a whole.

Table 3 Parameters at $4 \mathrm{~h}$ and their potential to discriminate between potential survivors and non-survivors

\begin{tabular}{lllllllll}
\hline Parameter & ROC AUC & $95 \% \mathrm{Cl}$ & $p$ value & Threshold & Sensitivity\% & $95 \% \mathrm{Cl}$ & Specificity\% & $95 \% \mathrm{Cl}$ \\
\hline Hct $(\%)$ & 0.93 & $0.779-1.0$ & 0.008 & $<43.5$ & 100 & $72-100 \%$ & 80 & $28-99 \%$ \\
Temperature $\left({ }^{\circ} \mathrm{C}\right)$ & 0.57 & $0.166-0.968$ & 0.673 & $>38.0$ & 60 & $15-95 \%$ & 83 & $52-98 \%$ \\
Lactate $(\mathrm{mmol} / \mathrm{L})$ & 0.68 & $0.319-1.0$ & 0.257 & $>1.49$ & 60 & $15-95 \%$ & 100 & $72-100 \%$ \\
Base excess & 0.56 & $0.264-0.863$ & 0.692 & $<2.20$ & 80 & $28-99 \%$ & 45 & $17-77 \%$ \\
pH & 0.59 & $0.266-0.916$ & 0.571 & $>7.43$ & 40 & $5-85 \%$ & 82 & $48-98 \%$ \\
Glucose & 0.80 & $0.554-1.0$ & 0.066 & $>15.5$ & 60 & $15-95 \%$ & 90 & $56-100 \%$ \\
IL-1a (pg/ml) & 0.58 & $0.237-0.926$ & 0.610 & $>57.2$ & 40 & $5-85 \%$ & 91 & $59-100 \%$ \\
IL-1b (pg/ml) & 0.56 & $0.194-0.933$ & 0.692 & $>244$ & 60 & $15-95 \%$ & 82 & $48-98 \%$ \\
IL-6 (pg/ml) & 0.67 & $0.326-1.0$ & 0.282 & $>2775$ & 60 & $15-95 \%$ & 91 & $59-100 \%$ \\
IL-10 (pg/ml) & 0.60 & $0.217-0.983$ & 0.533 & $>538$ & 60 & $15-95 \%$ & 91 & $59-100 \%$ \\
TNF-a (pg/ml) & 0.51 & $0.168-0.850$ & 0.955 & $<61$ & 40 & $5-85 \%$ & 82 & $48-98 \%$ \\
CXCL-1 (pg/ml) & 0.69 & $0.349-1.0$ & 0.234 & $>1885$ & 80 & $28-99 \%$ & 82 & $48-98 \%$ \\
MCP-1 (pg/ml) & 0.56 & $0.211-0.916$ & 0.692 & $>7.4$ & 60 & $15-95 \%$ & 82 & $48-98 \%$ \\
MIP-1 (pg/ml) & 0.82 & $0.604-1.0$ & 0.047 & $>211$ & 60 & $15-95 \%$ & 91 & $59-100 \%$ \\
BNP (pg/ml) & 0.76 & $0.521-1.0$ & 0.101 & $<0.15$ & 60 & $15-95 \%$ & 82 & $48-98 \%$
\end{tabular}

Animals were sacrificed at $4 \mathrm{~h}$ prior to fluid resuscitation and categorized according to their heart rate changes from baseline $(\geq 50 \mathrm{bpm}=$ predicted non-survivors; $<50 \mathrm{bpm}=$ predicted survivors). This cut-off was determined from $48 \mathrm{~h}$ mortality experiments (see main manuscript for details). Cl confidence interval, ROC AUC area under the receiver operator characteristics curve 
Tachycardia persisted in non-survivors despite aggressive fluid resuscitation suggesting that tachycardia was not only a result of insufficient preload, but likely also a sign of autonomic dysfunction. Significant differences in HRV were found between groups, suggesting autonomic dysfunction in septic animals, particularly in the non-survivors. The ratio between low frequency (LF) and high frequency (HF) heart rate variations was significantly decreased in non-survivors. While parasympathetic tone (HF variation of heart rate) dominates over sympathetic tone (LF variation) under physiological conditions, LF/ HF variability could be lost during critical illness. As described in patients with sepsis [23] and heart failure [24], autonomic regulatory dysfunction can lead to a low LF/HF ratio despite a high level of sympathetic activation. In agreement with our preclinical study, a low LF/HF ratio was associated with an increased risk of death in septic patients [25-27]. Our results are also comparable to sepsis studies in rodents [28, 29] and humans [30], in which similar results of reduced HRV have been observed. The interruption of specific feedback mechanisms between the autonomic nervous system and the heart is termed uncoupling of the components [31]; autonomic dysfunction may thus represent a potential mechanism resulting in death. Further clinical studies on heart rate and heart rate variability in septic patients are needed to better discriminate between compensatory tachycardia (adaptive) and non-compensatory tachycardia (maladaptive), and to understand when tachycardia should be treated with beta-blocking agents.

The stability of gas exchange and metabolic acidaemia at the $24 \mathrm{~h}$ timepoint suggest that respiratory failure or renal dysfunction is not directly responsible for mortality in this model. The absence of elevated troponin levels suggests that myocardial injury was also not a hallmark. However, elevation in BNP at this timepoint is indicative of myocardial dysfunction and mirrors clinical findings in patients with established sepsis, particularly in those with a poor prognosis [32-35]. This finding implies heart failure may be an important mechanism underlying death.

Mean cytokine levels were non-significantly higher in predicted non-survivors, albeit with high variability. Cytokine levels had normalized by $24 \mathrm{~h}$ in survivors, which concurrent with features of clinical recovery of signs. A similar pattern was reported by Recknagel et al. in their rat model of polymicrobial sepsis [36].

\section{Limitations of the study}

We cannot report on circulating catecholamine levels, as this model does not allow repetitive and/or non-stressful blood sampling in awake animals. The 4 and $24 \mathrm{~h}$ blood samples were collected after prior surgical exposure of the carotid artery, which would invalidate catecholamine measurements at these particular timepoints.

All animals received the same fluid protocol. As shown in Table 1, predicted sepsis non-survivors had an increased hematocrit compared to sepsis survivors and sham animals, which may indicate intravascular hypovolemia. However, administration of larger amounts of intravenous fluids could be harmful for potential survivors and sham animals [5]. Fluid administration titrated to physiological parameters might offer further improvement of the model. However, heart rate alone is not useful to guide fluid resuscitation, as non-compensatory tachycardia can persist after fluid resuscitation [5].

HRV is influenced by heart rate [37] and respiration rate [38], which both changed substantially in septic rats, particularly in the non-survivors. We did not perform any 
intervention to directly test whether the recorded frequency bands (LF or HF) correlate with sympathetic and parasympathetic responses in our model. Furthermore, there was no recovery period between telemetry implantation and baseline measurements. While surgery and anesthesia have likely influenced heart rate and heart rate variability, this would affect all experimental groups. Potentially, the differences found in our study could be even more pronounced if there was a recovery phase between telemetry implantation and induction of peritonitis. However, a recovery phase would also increase the risk of wound infections or adverse effects of empirical antibiotic therapy that could also affect the results of the study.

\section{Conclusions}

We describe a fluid-resuscitated rat model of abdominal sepsis with a representative sepsis phenotype and long-term mortality. This model represents not only an alternative to the standard mouse CLP model, but one more representative of human sepsis with respect to physiological, metabolic, and transcriptome changes [39, 40]. Telemetry-monitored changes in heart rate as early as $4 \mathrm{~h}$ after the septic insult predicted death with good sensitivity and specificity. Previous studies have demonstrated that autonomic dysfunction occurs during sepsis. The HRV analysis of this study supports this finding, but the results must be interpreted with care due to several limitations. Whether autonomic dysfunction during sepsis is a mechanism leading to death requires further mechanistic studies with a special focus on HRV. Further investigations of adaptive changes in potential survivors and mechanisms of death in potential non-survivors are possible with this model. It will also allow testing of novel treatment to assess their beneficial and harmful effects in predicted sepsis non-survivors and survivors.

\section{Abbreviations}

AUC: Area under the curve; BNP: B-type natriuretic peptide; BPM: Beats per minute; Cl: Confidence interval; CLP: Cecal ligation and puncture; ECG: Electrocardiogram; HR: Heart rate; LPS: Lipopolysaccharide; ROC: Receiver operating characteristicS; SD: Standard deviation; SEM: Standard error of the mean

\section{Acknowledgements}

We thank Professor Rudolf Zbinden (Institute for Medical Microbiology, University of Zurich, Zurich, Switzerland) for performing the microbiological tests.

Funding

This work was supported by a research grant from the Swiss Society of Intensive Care Medicine (2010), an Established Investigator Award from the European Society of Intensive Care Medicine (2011), and a Research Grant from the Privatdozenten-Stiftung of the University Zurich (2016), all awarded to AR.

\section{Authors' contributions}

$A R, V J$, MArrigo, MArras, MS, GS, DS, and DB helped to design the work. AR, VJ, MArrigo, CS, FH, and GS helped in the acquisition of data. $A R, V J$, and $B S$ contributed to the statistical analysis of data. AR, VJ, and MArrigo helped to draft the manuscript. CS, FH, MArras, MS, DS, and DB critically reviewed the manuscript for important intellectual content. All authors gave the final approval of the version submitted for publication.

Ethics approval

All protocols were approved by the Animal Experimentation Committee at the Veterinary Office of the Canton of Zurich, Switzerland. Principles of the 3Rs were implemented.

Consent for publication

Not applicable

\section{Competing interests}

AR has received lecture fees for talks on esmolol, a short-acting beta-blocker, from OrphaSwiss GmbH and AMOMED, the distributors of nalbuphine in Switzerland and Europe. MS is a UK National Institute of Health Research Senior Investigator.

DRS' academic department is/has been receiving grant support from the Swiss National Science Foundation, Berne, Switzerland, the Ministry of Health (Gesundheitsdirektion) of the Canton of Zurich, Switzerland for Highly Specialized 
Medicine, the Swiss Society of Anesthesiology and Reanimation (SGAR), Berne, Switzerland, the Swiss Foundation for Anesthesia Research, Zurich, Switzerland, Bundesprogramm Chancengleichheit, Berne, Switzerland, CSL Behring, Berne, Switzerland, Vifor SA, Villars-sur-Glâne, Switzerland. DRS was the chair of the ABC Faculty and is the co-chair of the ABC-Trauma Faculty, managed by Physicians World Europe GmbH, Mannheim, Germany and sponsored by unrestricted educational grants from Novo Nordisk Health Care AG, Zurich, Switzerland, CSL Behring GmbH, Marburg, Germany and LFB Biomédicaments, Courtaboeuf Cedex, France.

In the past 5 years, DRS has received honoraria or travel support for consulting or lecturing from Abbott AG, Baar, Switzerland, AMGEN GmbH, Munich, Germany, AstraZeneca AG, Zug, Switzerland, Baxter AG, Volketswil, Switzerland, Baxter S.p.A., Roma, Italy, Bayer, Zürich, Switzerland and Berlin, Germany, B. Braun Melsungen AG, Melsungen, Germany, Boehringer Ingelheim (Schweiz) GmbH, Basel, Switzerland, Bristol-Myers-Squibb, Rueil-Malmaison Cedex, France and Baar, Switzerland, CSL Behring GmbH, Hattersheim am Main, Germany and Berne, Switzerland, Curacyte AG, Munich, Germany, Daiichi Sankyo (Schweiz) AG, Thalwil, Switzerland, Danube University of Krems, Austria, Ethicon Biosurgery, Sommerville, New Jersey, USA, Fresenius SE, Bad Homburg v.d.H., Germany, Galenica AG, Bern, Switzerland (including Vifor SA, Villars-sur-Glâne, Switzerland), GlaxoSmithKline GmbH \& Co. KG, Hamburg, Germany, Janssen-Cilag, Baar, Switzerland and Beerse, Belgium, LFB Biomédicaments, Courtaboeuf Cedex, France, Merck Sharp \& Dohme AG, Luzern, Switzerland, Novo Nordisk A/S, Bagsvärd, Denmark, Octapharma AG, Lachen, Switzerland, Organon AG, Pfäffikon/SZ, Switzerland, PAION Deutschland GmbH, Aachen, Germany, Pharmacosmos A/S, Holbaek, Denmark, Photonics Healthcare B.V., Utrecht, Netherlands, ratiopharm Arzneimittel Vertriebs-GmbH, Vienna, Austria, Roche, Reinach, Switzerland, Sarstedt AG \& Co., Sevelen, Switzerland, Schering-Plough International, Inc., Kenilworth, New Jersey, USA, Tem International GmbH, Munich, Germany, Verum Diagnostica GmbH, Munich, Germany, US Department of Defense, Washington, USA, Vifor Pharma, Munich, Germany, Vienna, Austria and St. Gallen, Switzerland.

For the remaining authors, no relevant conflicts of interest or sources of funding were declared.

\section{Publisher's Note}

Springer Nature remains neutral with regard to jurisdictional claims in published maps and institutional affiliations.

\section{Author details}

${ }^{1}$ Institute of Anesthesiology, University and University Hospital Zurich, Raemistrasse 100, 8091 Zurich, Switzerland. ${ }^{2}$ Inflammation Research Unit, Division of Internal Medicine, University and University Hospital Zurich, Raemistrasse 100, CH 8091 Zurich, Switzerland. ${ }^{3}$ Clinic for Cardiology, University Heart Centre, University and University Hospital Zurich, Raemistrasse 100, CH 8091 Zurich, Switzerland. ${ }^{4}$ Department of Surgery, University and University Hospital Zurich, Raemistrasse 100, CH 8091 Zurich, Switzerland. ${ }^{5}$ Department of Biostatistics at Epidemiology, Biostatistics and Prevention Institute, University of Zurich, Hirschengraben 84, 8001 Zurich, Switzerland. ${ }^{6}$ Bloomsbury Institute of Intensive Care Medicine, Division of Medicine, University College London, Gower Street, London WC1E 6BT, UK.

\section{Received: 23 January 2018 Accepted: 19 July 2018}

\section{Published online: 20 August 2018}

\section{References}

1. Singer M, Deutschman CS, Seymour CW, Shankar-Hari M, Annane D, Bauer M et al (2016) The third international consensus definitions for sepsis and septic shock (Sepsis-3). JAMA 315:801-810

2. Angus DC, Lindle-Zwirble WT, Clermont G, Carcillo J, Pinsky MR (2001) Epidemiology of severe sepsis in the United States: analysis of incidence, outcome, and associated costs of care. Crit Care Med 29:1303-1310

3. Vincent JL, Marshall JC, Namendys-Silva SA, Francois B, Martin-Loeches I, Lipman J et al (2014) Assessment of the worldwide burden of critical illness: the intensive care over nations (ICON) audit. Lancet Respir Med 2:380-386

4. Angus DC, van der Poll T (2013) Severe sepsis and septic shock. N Engl J Med 369:840-851

5. Rudiger A, Dyson A, Felsmann K, Carre JE, Taylor V, Hughes S et al (2013) Early functional and transcriptomic changes in the myocardium predict outcome in a long-term rat model of sepsis. Clin Sci 124:391-401

6. Kilkenny C, Browne WJ, Cuthill IC, Emerson M, Altman DG (2010) Improving bioscience research reporting: the ARRIVE guidelines for reporting animal research. PLoS Biol 8:e1000412

7. Reynolds P, Wall P, van Griensven M, McConnell K, Lang C, Buchman T (2012) Shock supports the use of animal research reporting guidelines. Shock 38:1-3

8. Dyson A, Rudiger A, Singer M (2011) Temporal changes in tissue cardiorespiratory function during faecal peritonitis. Intensive Care Med 37:1192-1200

9. Arulkumaran N, Sixma ML, Jentho E, Ceravola E, Bass PS, Kellum JA et al (2017) Sequential analysis of a panel of biomarkers and pathologic findings in a resuscitated rat model of sepsis and recovery. Crit Care Med 45:e821-e830

10. Jeger V, Arrigo M, Hildenbrand FF, Müller D, Jirkof P, Hauffe T et al (2017) Improving animal welfare using continuous nalbuphine infusion in a long-term rat model of sepsis. Intensive Care Med Exp 5:17

11. Jeger V, Hauffe T, Nicholls-Vuille F, Bettex D, Rudiger A (2016) Analgesia in clinically relevant rodent models of sepsis. Lab Anim 50:418-426

12. Hollenberg SM, Dumasius A, Easington C, Colilla SA, Neumann A, Parrillo JE (2001) Characterization of a hyperdynamic murine model of resuscitated sepsis using echocardiography. Am J Respir Crit Care Med 164:891-895

13. Task Force of the European Society of Cardiology and the North American Society of Pacing and Electrophysiology (1996) Heart rate variability - standards of measurement, physiological interpretation, and clinical use. Eur Heart J 17:354-381

14. Rowan WH, Campen MJ, Wichers LB, Watkinson WP (2007) Heart rate variability in rodents: uses and caveats in toxicological studies. Cardiovasc Toxicol 7:28-51

15. Magder SA (2012) The ups and downs of heart rate. Crit Care Med 40:239-245

16. Cook S, Togni M, Schaub MC, Wenaweser P, Hess OM (2006) High heart rate: a cardiovascular risk factor? Eur Heart J 27:2387-2393 
17. Parker MM, Shelhamer JH, Natanson C, Alling DW, Parrillo JE (1987) Serial cardiovascular variables in survivors and nonsurvivors of human septic shock: heart rate as an early predictor of prognosis. Crit Care Med 15:923-929

18. Azimi G, Vincent J-L (1986) Ultimate survival from septic shock. Resuscitation 14:245-253

19. Sander O, Welters ID, Foex P, Sear JW (2005) Impact of prolonged elevated heart rate on incidence of major cardiac events in critically ill patients with a high risk of cardiac complications. Crit Care Med 33:81-88 discussion 241-242

20. Domizi R, Calcinaro S, Beilstein C, Boerma C, Ciche J-D, D'Egidio AD et al (2014) Relationship between catecholamine dose, tachycardia and outcome in septic shock: a multicentre evaluation (meeting abstract 0300). Intensive Care Med 40:589

21. Lukaszewicz AC, Payen D (2010) The future is predetermined in severe sepsis, so what are the implications? Crit Care Med 38:S512-S517

22. Singer M, Glynne P (2005) Treating critical illness: the importance of first doing no harm. PLoS Med 2:e167

23. Annane D, Trabold F, Sharshar T, Jarrin I, Blanc AS, Raphael J-C et al (1999) Inappropriate sympathetic activation at onset of septic shock: a spectral analysis approach. Am J Respir Crit Care Med 160:458-465

24. van de Borne P, Montano N, Pagani M, Oren R, Somers VK (1997) Absence of low-frequency variability of sympathetic nerve activity in severe heart failure. Circulation 95:1449-1454

25. Korach M, Sharshar T, Jarrin I, Fouillot JP, Raphael JC, Gajdos P et al (2001) Cardiac variability in critically ill adults: influence of sepsis. Crit Care Med 29:1380-1385

26. Winchell RJ, Hoyt DB (1996) Spectral analysis of heart rate variability in the ICU: a measure of autonomic function. J Surg Res 63:11-16

27. de Castilho FM, Ribeiro ALP, da Silva JLP, Nobre V, de Sousa MR (2017) Heart rate variability as predictor of mortality in sepsis: a prospective cohort study. PLoS One 12:e0180060

28. Pancoto JA, Correa PB, Oliveira-Pelegrin GR, Rocha MJ (2008) Autonomic dysfunction in experimental sepsis induced by cecal ligation and puncture. Auton Neurosci 138:57-63

29. Zila I, Mokra D, Kopincova J, Kolomaznik M, Javorka M, Calkovska A (2015) Heart rate variability and inflammatory response in rats with lipopolysaccharide-induced endotoxemia. Physiol Res 64(Suppl 5):S669-S676

30. Tateishi Y, Oda S, Nakamura M, Watanabe K, Kuwaki T, Moriguchi T et al (2007) Depressed heart rate variability is associated with high IL-6 blood level and decline in the blood pressure in septic patients. Shock 28:549-553

31. Buchman TG (2002) The community of the self. Nature 420:246-251

32. Charpentier J, Luyt C-E, Fulla Y, Visonneau C, Cariou A, Grabar S et al (2004) Brain natriuretic peptide: a marker of myocardial dysfunction and prognosis during severe sepsis. Crit Care Med 32:660-665

33. Rudiger A, Gasser S, Fischler M, Hornemann T, Von Eckardstein A, Maggiorini M (2006) Comparable increase of Btype natriuretic peptide and amino-terminal pro-B-type natriuretic peptide levels in patients with severe sepsis, septic shock, and acute heart failure. Crit Care Med 34:2140-2144

34. Post F, Weilemann LS, Messow CM, Sinning C, Munzel T (2008) B-type natriuretic peptide as a marker for sepsisinduced myocardial depression in intensive care patients. Crit Care Med 36:3030-3037

35. Hayase N, Yamamoto M, Asada T, Isshiki R, Yahagi N, Doi K (2016) Association of heart rate with N-terminal pro-Btype natriuretic peptide in septic patients: a prospective observational cohort study. Shock 46:642-648

36. Gonnert FA, Recknagel P, Seidel M, Jbeily N, Dahlke K, Bockmeyer CL et al (2011) Characteristics of clinical sepsis reflected in a reliable and reproducible rodent sepsis model. J Surg Res 170:e123-e134

37. Mangin L, Swynghedauw B, Benis A, Thibault N, Lerebours G, Carre F (1998) Relationships between heart rate and heart rate variability: study in conscious rats. J Cardiovasc Pharmacol 32:601-607

38. Dick TE, Hsieh YH, Dhingra RR, Baekey DM, Galan RF, Wehrwein E et al (2014) Cardiorespiratory coupling: common rhythms in cardiac, sympathetic, and respiratory activities. Prog Brain Res 209:191-205

39. Zolfaghari PS, Pinto BB, Dyson A, Singer M (2013) The metabolic phenotype of rodent sepsis: cause for concern? Intensive Care Med Exp 1:25

40. Efron PA, Mohr AM, Moore FA, Moldawer LL (2015) The future of murine sepsis and trauma research models. J Leukoc Biol 98:945-952

\section{Submit your manuscript to a SpringerOpen ${ }^{\circ}$ journal and benefit from:}

- Convenient online submission

- Rigorous peer review

- Open access: articles freely available online

- High visibility within the field

- Retaining the copyright to your article

Submit your next manuscript at $\boldsymbol{\nabla}$ springeropen.com 